\title{
Designing User Interfaces for Social Media Driven Digital Preservation and Information Retrieval
}

\author{
Dimitris Spiliotopoulos ${ }^{1,2}$, Efstratios Tzoannos ${ }^{1}$, Pepi Stavropoulou ${ }^{2}$, \\ Georgios Kouroupetroglou ${ }^{2}$, and Alexandros Pino ${ }^{3}$ \\ ${ }^{1}$ Athens Technology Centre, Chalandri, Athens, Greece \\ \{d.spiliotopoulos, e.tzoannos\} @atc.gr \\ ${ }^{2}$ National and Kapodistrian University of Athens, \\ Dep. of Informatics and Telecommunications \\ ${ }^{3}$ National and Kapodistrian University of Athens, Accessibility Unit, \\ Panepistimiopolis, Ilissia, 15784, Athens, Greece \\ \{pepis, koupe, pino\} @di.uoa.gr
}

\begin{abstract}
Social Media provide a vast amount of information identifying stories, events, entities that play the crucial role of shaping the community in an everyday heavy user involvement. This work involves the study of social media information in terms of type (multimodal: text, video, sound, picture) and role players (agents, users, opinion leaders) and the potential of designing accessible, usable interfaces that integrate that information. This case examines the design of a user interface that uses an underlying engine for modality components (plain text, sound, image, video) analysis, social media crawling, contextual search fusion and semantic analysis. The interface is the only point of user interaction to the world of knowledge. This work reports on the usability and accessibility methods and concerns for the user requirements phase and the design control and testing. The findings of the pilot user testing and evaluation provide indications on how the semantic analysis of the social media information can be integrated to the design methodologies for user interfaces resulting in maximization of user experience in terms of social information involvement.
\end{abstract}

Keywords: social media, user interface design, user enablement.

\section{Introduction}

Social media content is collected from several social media, from users enabled by mobile devices, web pages, smart cameras, etc. Social media are becoming more and more pervasive in all areas of life. The major social media (Twitter, Facebook, Youtube, Flickr and others) are the hubs of the social information, a constant stream of targeted content, enabling people to report, comment, converse while engaged in their everyday activities, like work, study, travel, leisure. The people are enabled to provide content that is highly contextualized, user specific, yet responding to the society events and ideas in a rapid manner [2]. The citizen involvement is now so 
huge that we have come to the point where the social media information creation and communication now characterizes the information society. It is therefore no surprise that searching the web returns more social media hits than web pages.

As part of the greater approach of the EU ICT ARCOMEM ${ }^{1}$ project the design of information retrieval interfaces for the following two domains has been undertaken:

- Socially contextualized Broadcaster/Media archiving

- Socially-aware federated political archiving

The aim of the interfaces is to encourage the user involvement not only on content creation and communication (the purpose of social media) but also on content analysis, preservation and reuse for identifying the major events as they happen, analyze the social input in its content (opinion and sentiment) and re-involve the users in an iterative idea/opinion/evaluation, a socially contextual process [3].

\section{Design Considerations}

The requirements for both aforementioned domains are similar as a generic concept. The high-level requirements were selected from groups of users from broadcaster organizations and political bodies. The contextual framework for the information retrieval specifications has been identified and described by a list of generic, domain relevant and domain specific terms. The terms include semantic entities/events/topics, sentiment and opinions, social and demographic information, contextual information (actors, influencers) from a range of social media and social media-related sources, such as Blogs, Microblogs, Wikis, Social Networks, Video/Photo/Music/Audio Networks, Discussion groups, Social Bookmarks [1], [4 - 6].

Four social media were selected for this phase of the work, namely Twitter, Flickr, Youtube, Facebook, spanning from text-only heavily opinionated (Twitter) to multimedia oriented ways of integrating the user generated content to the ICT life (Flickr, Youtube). For the pilot design and testing, the socially-aware federated political search and retrieval interface prototype was used.

Accessibility is major concern and it is manifested in the form of universal design that, in our case, dictates that the UI should adhere to principles such as simplicity, flexibility, effort considerations. The integration of opinions, entities, trends, authors and statistics from social networks should be seamless in order to be usable.

Iterative design process from the start to the implementation of the first prototype was followed from the initial design requirements gathering (made via wire-framing) to the mockup user environment, to the online prototype A. Mockups of the tool pages were constructed keeping in mind the three types of information from the functional specifications (Search filtering, Core content, Social content) and the nonfunctional specifications. The initial experimentation was on the observation of the user perception of processed content (filters, tag clouds, paths), direct content (item descriptions, authors, dates, type of modality) and social content (opinions, trends,

${ }^{1}$ ARCOMEM: Archive Communities Memories, www . arcomem. eu, FP7-ICT-270239. 
semantics, entities, events). Based on the above, low fidelity mockups were created for the web retrieval interface. Those were subsequently evaluated for the core functionalities (filtering of results, follow-up search, results visualization) as well as usability (user approach to semantic search, information load, user effort, acceptance). An early prototype was build based on the previous feedback and was informally evaluated by usability experts. That intermediate evaluation was a short approach to applying generic accessibility criteria to the early prototype. In our case, universal design principles were applied for the scope of providing a solid basis for extending the interface future versions to multitouch devices (namely iOS) as well as voice interfaces, although the latter is not in the specification. The result of the above approaches was the implementation of the prototype user interface that was evaluated by expert and non-expert users as described in the following section.

\section{Experiments}

Three distinct experiments based on the initial information derived from the user requirements were set up. The purpose was to refine the state-of-the-art general methods for interface design with social content driven methods of retrieving, navigating, and optimizing information in order to maximize the user engagement and experience.

The first experiment was to evaluate the impact of social media feedback to the search results of the interfaces using guided sorting and filtering per media category. The users have been asked to perform searches based on pre-determined scenarios, and the returned results and dynamic filtering were evaluated for their social media source impact. Between the search results, the users were 3 times more likely to select an item if it had an opinion banner (top-right on the item in Fig. 1). Also, most of the users reported their selections to view specific items were influenced by the trending information (bottom-right in Fig. 1), while the social network source information (bottom-left in Fig. 1) did not influence their selections either positively or negatively.

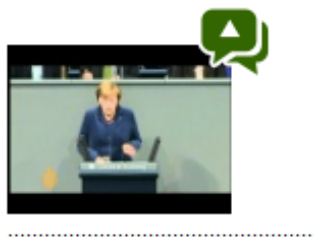

Merkel says Greece has chance to overcome crisis

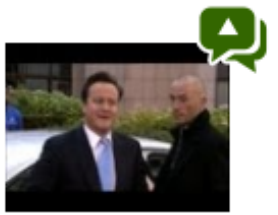

Greece Strikes Deal to End Financial Crisis

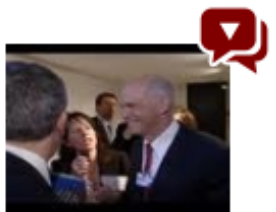

Papandreou's referendum is a gamble too far
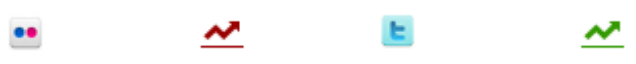

\section{$\bullet$}

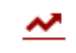

Fig. 1. Social network source, opinion and trending information

The second experiment investigated how sentiment is transferred from the information content (social media opinion mining) to the target users and how that sentiment (opinion) triggers the follow-up searches (involvement) and the intended results from the user perspective (contextual search validation). The users were asked 
to randomly perform three initial searches and then follow up their topic of interest. The subjects followed their topics mostly based on entities that carried negative or positive opinions. An important observation was that the subjects' navigation was governed by the amount of opinions about entities or events, while more than $80 \%$ of the opinions for the entities were accessed and viewed.

The final experiment was the usability evaluation. One of the main requirements was to measure the impact of the social media derived content (sentiment, modality, topic/event identification) to the user communication and engagement as a parameter for acceptance. Options for facets, filtering of emotions and modality selections were presented to the users and their feedback was measured accordingly. The subjects reported that the connections between the search results and the associated entities (people, places) were on target. The items that did not carry many opinions were quickly passed through by the subjects in order to find items that were very heavily commented upon. The acceptance of the approach was very high, however specific features were requested, mainly timelines for opinion trends. Another conclusion of the logged navigation entries suggests that the ranking of results should be based on amount of opinions associated with them rather than trends or social network sources.

\section{Conclusion}

Based on the results of the experimenting with fusing and visualizing the social content with semantically driven context sensitive information processing, the classic user interface design methods have been extended to maximize the social impact, encourage user responses and even indicate potential events and associated opinions as very important candidates for policy making. Further work is currently underway for the training of the user groups in setting up and engaging to the socially-aware information retrieval and archiving processes. The results of this work are expected to enhance the design of the user interface to support and sustain a socially driven user involvement into the vast amount of information.

\section{References}

1. Agarwal, et al.: Identifying the influential bloggers in a community. In: Proc. of the First International Conference on Web Search and Web Data Mining (WSDM 2008) (2008)

2. Agichtein, et al.: Finding high quality content in social media, with an application to community-based question answering. In: Proc. of ACM WSDM, pp. 183-194. ACM Press, Stanford (2008)

3. Anagnostopoulos, et al.: Influence and correlation in social networks. In: Proc. of the 14th ACM SIGKDD, Int. Conf. on Knowledge Discovery and Data Mining (KDD 2008) (2008)

4. Pang, Lee: Opinion mining and sentiment analysis: Foundations and Trends in Information Retrieval 2(1-2), 1-135 (2008)

5. Weining, et al.: Image retrieval by emotional semantics: A study of emotional space and feature extraction 4, 3534-3539 (2006)

6. Zontone, et al.: Image diversity analysis: Context, opinion and bias. In: The First International Workshop on Living Web: Making Web Diversity a true asset, vol. 515, CEUR-WS (October 2009) 